\title{
Ciliated muconodular papillary tumour of the lung mimicking mucinous adenocarcinoma: a case report and literature review
}

\author{
Florence MF Cheung * 1, MB, BS, FHKAM (Pathology), J Guan'1, MD, PhD, QG Luo², MD, \\ Alan DL Sihoe ${ }^{2}$, MBBChir, FHKAM (Surgery), XP Shen ${ }^{3}$, MD \\ ${ }^{1}$ Department of Pathology, ${ }^{2}$ Department of Thoracic Surgery, ${ }^{3}$ Department of Radiology, University of Hong Kong-Shenzhen Hospital, \\ Shenzhen, Guangdong, China \\ *Corresponding author: fmfcheung@gmail.com \\ Hong Kong Med J 2019;25:71-3 \\ https://doi.org/10.12809/hkmj165032
}

\section{Case report}

Solitary lung nodules of $<3 \mathrm{~cm}$ in diameter within the lung parenchyma and with no other abnormalities are often picked up incidentally during routine radiographic imaging. The incidence of cancer for such nodules has been estimated to be $10 \%$ to $70 \%$. Management strategy depends on the clinical probability of cancer; nodule size, features, and growth rate ascertained by radiology; and the surgical risk to the patient. We report a case of such a nodule revealed by radiology and suspicious for malignancy. Subsequent excision and pathological examination revealed unexpected findings.

The index patient was a 61-year-old Chinese man from Northern China with history of laryngeal cancer treated successfully by local surgery and radiotherapy 6 years prior to present admission. Routine chest computed tomography (CT) scan revealed a peripheral lung nodule $9 \mathrm{~mm}$ in diameter in the right lower lobe. Follow-up CT scan 1 year later revealed minimal increase in size to $10 \mathrm{~mm}$ and the patient was referred to the Department of Thoracic Surgery, University of Hong KongShenzhen Hospital in November 2015 for further treatment. The patient was a former chronic smoker for more than 10 years (10 cigarettes/day) but had stopped smoking upon diagnosis of laryngeal cancer.

Physical examination of the patient was unremarkable. High-resolution CT scan confirmed the presence of a peripheral lung nodule in his right lower lobe lateral-basal segment that was suspicious for malignancy. It measured $12 \mathrm{~mm}$ in diameter and had a spiculated border with a central cavity (Fig a). Another high-density 2-mm nodule was present in the right upper lobe subpleural region associated with apical fibrosis. There was pleural thickening and mildly increased peripheral lung markings in bilateral lower lobes. The peribronchiolar and hilar lymph nodes were not enlarged. After further examination and assessment of the surgical risk, video-assisted thoracoscopy was decided, with patient consent.

During video-assisted thoracoscopy, wedge excision of the nodule was done. Intra-operative frozen section consultation revealed a $10-\mathrm{mm}$ papillary glandular tumour $8 \mathrm{~mm}$ away from the pleura. There was profuse mucin production and intra-alveolar extension suspicious for mucinous adenocarcinoma. Right lower lobectomy was subsequently performed, and the patient had an uneventful recovery. Microscopic examination of formalin-fixed paraffin-embedded sections from the nodule showed an arborising papillary tumour (Fig b) surrounded by intra-alveolar mucin. There was extension along the alveolar lining at its periphery (Fig b, inset) simulating 'lepidic spread' of adenocarcinoma. The papillary structures (Fig c and d) consisted of fibrous cores covered by single to multiple layers of mucin-secreting and ciliated (Fig d, inset) columnar cells and basal cells. There was focal tumour necrosis, rare mitoses, and mild nuclear atypia. Mucin and inflammatory cells filled the central cystic space. Origin from a dilated terminal bronchiole could be traced (Fig e). A batch of immunohistochemical studies showed $\mathrm{CK} 7+1$ CK20- tumour cells mostly negative for thyroid transcription factor-1 except at the periphery, suggestive of residual alveolar lining cells. Monoclonal carcinoembryonic antigen highlighted the mucinous cells and p63 stained the basal cells. Proliferative index by Ki67 was low (5\%). The overall picture was consistent with ciliated muconodular papillary tumour (CMNPT) of the lung. Examination of the lobectomy specimen showed focal peribronchiolar fibrosis compatible with the effect of smoking. ${ }^{1}$ These foci often contained peribronchiolar metaplasia featuring ciliated and mucinous columnar cells (Fig $\mathrm{f}$ ) occasionally forming small papillae (Fig $\mathrm{f}$, inset). The 2-mm lesion in the right upper lobe was a fibrotic nodule. The patient was well 1 year after surgery.

\section{Discussion}

The term CMNPT of the lung was first used by Ishikawa in 2002 to describe a $1.5-\mathrm{cm}$ peripheral lung nodule consisting of ciliated columnar 


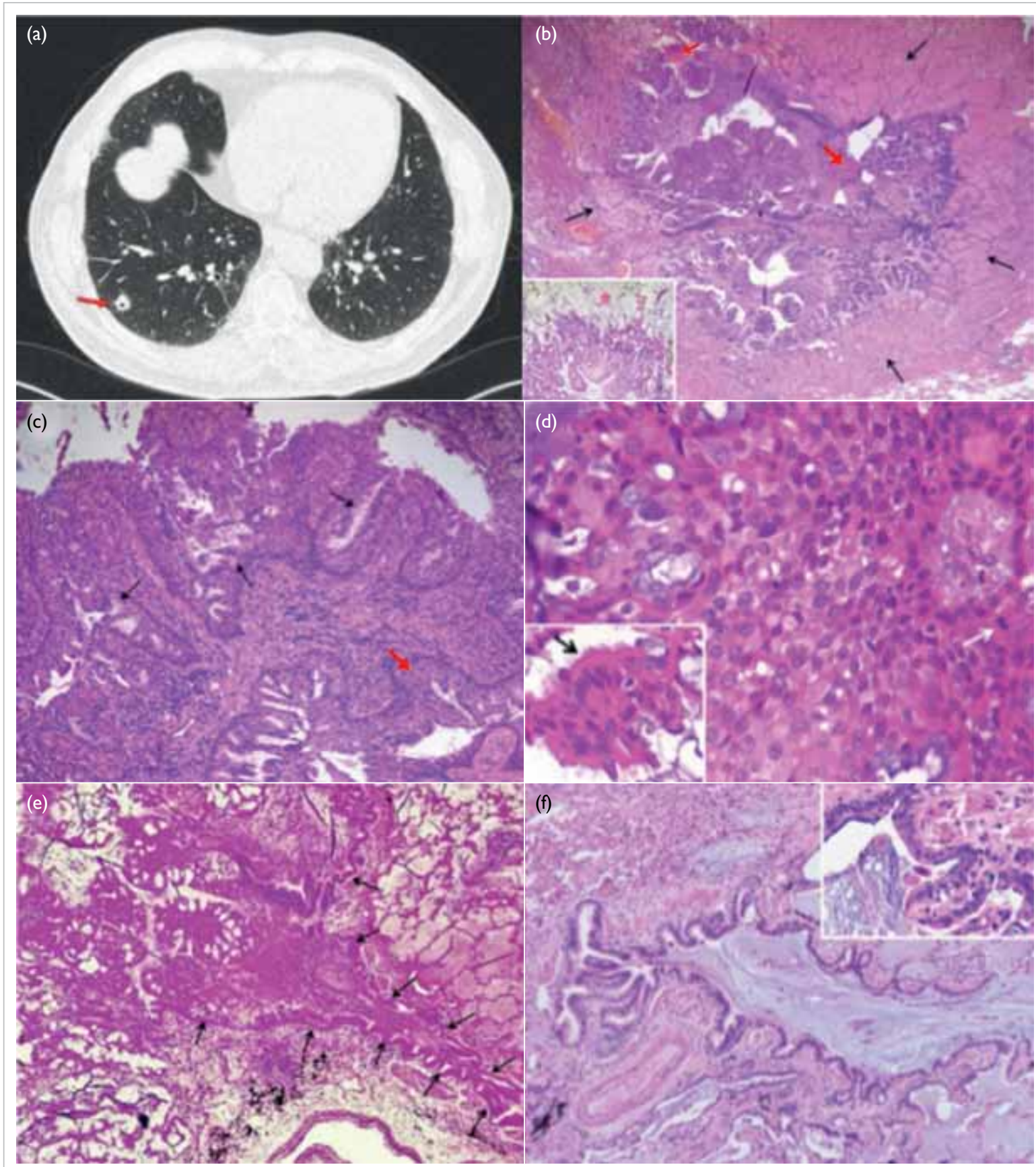

FIG. Radiological and pathological findings of the right lower lobe of the lung. (a) High-resolution computed tomography shows right lower lobe lung nodule (red arrow) with spiculated border and central cavity. (b) Lung nodule surrounded by a ring of intra-alveolar mucin (black arrows). Scattered tumour necrosis is seen (red arrows). Inset: Frozen section highlights intraalveolar growth pattern and central cystic space (haematoxylin \& eosin [H\&E], x 10). (c) Papillary structures consist of multilayered mucin-secreting columnar cells (black arrows) and basal cells (red arrow) on fibrous cores (H\&E, $\times 40)$. (d) Basal cells have mildly atypical nuclei with prominent nucleoli and rare mitosis (white arrow). Inset: ciliated cells (black arrow) (H\&E, $\times 400)$. (e) Origin of the tumour from a dilated respiratory bronchiole can be demonstrated (outline is black-arrowed) [H\&E, $\times 20]$. (f) Section from adjacent lung shows peribronchiolar mucociliated metaplasia (inset: $\mathrm{H} \& \mathrm{E}, \times 400$ ) of a terminal bronchiole extending into alveolar wall $(\mathrm{H} \& \mathrm{E}, \times 100)$

cells, mucous cells and basal cells with papillary glandular papillomas, peripheral pulmonary architecture. It was considered benign in view of papillary/glandular neoplasms with ciliated cells) indolent behaviour and bland-looking cells. Further supported this group of tumours as a specific entity reports by Ishikawa ${ }^{2}$ and other $\mathrm{s}^{3-7}$ of similar tumours that has not been included in the 2015 World Health under various names (eg, solitary peripheral ciliated Organization Classification of Lung Tumours. ${ }^{8}$ We 
searched the literature and reviewed 12 reports of 33 such tumours (online supplementary Appendix). Controversy exists whether CMNPT should be considered a benign tumour, a well-differentiated adenocarcinoma (in view of frequent intra-alveolar extension), or a spectrum of entities with possible progression. The consistent small size, slow growth rate, and lack of recurrence or metastasis after surgery support the benign nature of this tumour. Differentiation from mucinous adenocarcinoma is difficult for pathologists, especially during intraoperative frozen section, owing to the profuse mucin production and lepidic growth pattern. High-power examination revealing tripartite cell differentiation and lack of significant atypia in a clinically slowgrowing lung nodule should raise suspicion of CMNPT. Wedge excision with clear margin is the treatment of choice. Our findings concur with a previous report ${ }^{3}$ of tumour origin from the terminal bronchiole. The finding of co-existing peribronchiolar metaplasia with similar cell components as CMNPT in the rest of the lung is unique. This suggests progression of disease from smoking-induced metaplasia to neoplasia during the pathogenesis. Although chronic smoking was noted in most male patients with CMNPT (14 out of 16 with smoking history specified in the online supplementary Appendix), co-existing peribronchiolar metaplasia was only briefly mentioned in one report, ${ }^{4}$ probably owing to limited sampling in wedge excision for most tumours. Molecular analysis for $B R A F$ or EGFR mutations was not done in our case, because there was no therapeutic indication. Studies of CMNPT by Chuang et $\mathrm{al}^{5}$ and Lau et $\mathrm{al}^{6}$ yielded no $K R A S$ or EGFR mutation. In contrast, Kamata et $\mathrm{al}^{7}$ reported mutations involving EGFR, BRAF, PTEN11, CTNNB1, IDH1, and TP53 in Asian patients and Liu et $\mathrm{al}^{4}$ reported mutations involving $B R A F$ and $A K T 1$ in one non-Asian patient. Because CMNPT is commonly reported in patients from East Asia, more reports are expected when awareness of this entity is raised among pathologists in this region. The pathogenesis, molecular characteristics, and natural behaviour of CMNPT can be better defined when more data are available.

\section{Supplementary information}

Online supplementary information (Appendix) is available for this article at www.hkmj.org.

\section{Author contributions}

All authors had full access to the data, contributed to the study, approved the final version for publication, and take responsibility for its accuracy and integrity.

Concept or design: FMF Cheung.

Acquisition of data: All authors.

Analysis or interpretation of data: All authors.

Drafting of the manuscript: FMF Cheung.

Critical revision for important intellectual content: All authors.

\section{Acknowledgement}

The authors would like to thank Dr Siu-wah Pang for contributing to the diagnosis of this tumour.

\section{Conflicts of interest}

All authors have disclosed no conflicts of interest.

\section{Funding/support}

This research received no specific grant from any funding agency in the public, commercial, or not-for-profit sectors.

\section{Ethics approval}

This study was approved by the Ethics Committee of the University of Hong Kong-Shenzhen Hospital as original work with no infringement of personal privacy. The requirement for patient consent was waived by the Ethics Committee.

\section{References}

1. Katzenstein AL, Mukhopadhyay S, Zanardi C, Dexter E. Clinically occult interstitial fibrosis in smokers: classification and significance of a surprisingly common finding in lobectomy specimens. Hum Pathol 2010;41:31625.

2. Ishikawa $M$, Sumitomo $S$, Imamura $N$, et al. Ciliated muconodular papillary tumor of the lung: report of five cases. J Surg Case Rep 2016;2016.pii:rjw144.

3. Aida S, Ohara I, Shimazaki H, et al. Solitary peripheral ciliated glandular papillomas of the lung: a report of 3 cases. Am J Surg Pathol 2008;32:1489-94.

4. Liu L, Aesif SW, Kipp BR, et al. Ciliated muconodular papillary tumors of the lung can occur in Western patients and show mutations in $B R A F$ and $A K T 1$. Am J Surg Pathol 2016;40:1631-6.

5. Chuang HW, Liao JB, Chang HC, Wang JS, Lin SL, Hsieh PP. Ciliated muconodular papillary tumor of the lung: a newly defined peripheral pulmonary tumor with conspicuous mucin pool mimicking colloid adenocarcinoma: a case report and review of literature. Pathol Int 2014;64:352-7.

6. Lau KW, Aubry MC, Tan GS, Lim CH, Takano AM. Ciliated muconodular papillary tumor: a solitary peripheral lung nodule in a teenage girl. Hum Pathol 2016;49:22-6.

7. Kamata T, Sunami K, Yoshida A, et al. Frequent $B R A F$ or EGFR mutations in ciliated muconodular papillary tumors of the lung. J Thorac Oncol 2016;11:261-5.

8. Travis WD, Brambilla E, Burke AP, et al. WHO Classification of Tumours of the Lung, Pleura, Thymus and Heart. Lyon, France: International Agency for Research on Cancer; 2015. 